Scrape-Off-Layer Flow Studies in

Tokamaks: Final Report of LDRD Project 09-ERD-025

T. D. Rognlien, S. L. Allen, R. M. Ellis, G. D. Porter, S.

K. Nam, T. R. Weber, M. V. Umansky, J. Howard

November 22, 2011 
This document was prepared as an account of work sponsored by an agency of the United States government. Neither the United States government nor Lawrence Livermore National Security, LLC, nor any of their employees makes any warranty, expressed or implied, or assumes any legal liability or responsibility for the accuracy, completeness, or usefulness of any information, apparatus, product, or process disclosed, or represents that its use would not infringe privately owned rights. Reference herein to any specific commercial product, process, or service by trade name, trademark, manufacturer, or otherwise does not necessarily constitute or imply its endorsement, recommendation, or favoring by the United States government or Lawrence Livermore National Security, LLC. The views and opinions of authors expressed herein do not necessarily state or reflect those of the United States government or Lawrence Livermore National Security, LLC, and shall not be used for advertising or product endorsement purposes.

This work performed under the auspices of the U.S. Department of Energy by Lawrence Livermore National Laboratory under Contract DE-AC52-07NA27344. 


\title{
Scrape-Off-Layer Flow Studies in Tokamaks: Final Report of LDRD Project 09-ERD-025
}

\author{
T.D. Rognlien (PI), S.L. Allen, R.M. Ellis, G.D. Porter, \\ S.-K. Nam, T.R. Weber, and M.V. Umansky \\ Lawrence Livermore National Laboratory \\ Livermore, CA 94550 \\ J. Howard \\ The Australian National University \\ Canberra 0200, Australia
}

\begin{abstract}
A summary is given of the work carried out under the LDRD project 09-ERD-025 entitled Scrape-Off-Layer Flow Studies in Tokamaks. This project has lead to implementation of the new prototype Fourier Transform Spectrometer edge plasma flow diagnostic on the DIII-D National Fusion Facility at General Atomics, acquisition of carbon impurity concentration and flow data, and demonstration that the resulting data compare reasonably well with LLNL's edge plasma transport code UEDGE. Details of the work are contained in attached published papers, while the most recent results that are being written-up for publication are summarized in the report.
\end{abstract}




\section{Introduction}

Boundary plasma flows in tokamak fusion devices are key in determining the distribution of fuel and impurity ions, with tritium build-up in the walls an especially critical operational issue. The intrusion of impurity ions to the hot plasma core region can result in serious energy-loss owing to line radiation. However, flow diagnostic capability has been severely limited in fusion-relevant hot edge plasmas where Langmuir-type probes cannot withstand the high heat flux and traditional Doppler spectroscopy has limited resolution and signal strength. Thus, new edge plasma flow diagnostic capabilities need to be developed that can be used in existing and future devices such as ITER.

The understanding of such flows requires simulation with 2-dimensional transport codes owing to the geometrical complexity of the edge region in contact with material surfaces and the large number of interaction physical processes including plasma flow along and across the magnetic field, and coupling between impurity and neutral species. The characteristics of edge plasma flows are substantially affected by cross-magneticfield drifts $\left(\mathbf{E x B} / \mathrm{B}^{2}\right.$ and $\left.\mathbf{B} \times \nabla \mathrm{B} / \mathrm{B}^{2}\right)$, which are known to introduce substantial convergence difficulty for some cases. It is important that these difficulties be overcome so that drifts can be included in transport models, both for validation with existing data and for projection to future devices.

\section{Purpose of the LDRD Project}

To address this problem, a new, state-of-the-art diagnostic system has been developed, installed, and used to measure the flow profile of the carbon impurity ion species in the scrape-off layer (SOL) on the DIII-D National Fusion Facility tokamak. In parallel, detailed comparison of DIII-D data on poloidal distribution of impurity concentration and flow profiles has been obtained by the UEDGE transport code, along with the implementation and testing of advanced numerical algorithms. Validation of the UEDGE physics models with the new data will provide necessary confidence in predicting the SOL properties in future fusion devices, such as ITER.

\section{Approach}

A set of possible new diagnostic techniques for optically measuring the impurity flow were identified and prioritized as follows: high-throughput coherence imaging using

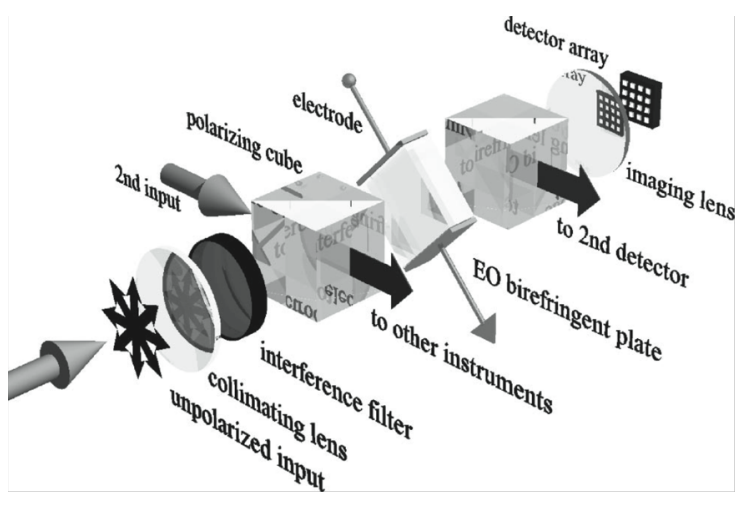

Fig. 1 Schematics of a FTS for analysis of the plasma flow velocity and temperature (from Ref. 1). a Fourier Transform Spectrometer (FTS) system [1], charge-exchange spectroscopy utilizing thermal hydrogen or a neutral hydrogen beam, and improved-resolution traditional spectroscopy. The FTS system was selected as first choice because of initial results shown in a non-tokamak device and because of the opportunity to collaborate with the world's leading authority on this technique, J. Howard of the Australian National University. If the initial FTS results appeared 
promising, work would then focus on obtaining high-quality data.

The modeling effort has utilized LLNL-based UEDGE 2D edge transport code. A focus of this work was to devise improved code performance that include the cross-field drift effects for a multi-species plasma model that includes individual charge states for each impurity ion. Validation of the impurity transport model of how impurities transport from the source region on the boundary throughout the SOL by comparison with DIII-D data for concentration level, and especially carbon density and flow data taken with the new FTS system.

\section{Technical Outcomes}

\section{Development/implementation/data for FTS system in DIII-D}

The LDRD-supported installation of the FTS system in DIII-D is described in detail in the attached publications [2,3], where the initial data obtained are also shown. The main purpose of this report is to highlight the most recent data and model comparisons that were presented at the APS-DPP Annual meeting in November 2012 [4] and are being written up for publication.

While the first set of data showed that the FTS system produced the type of signals anticipated, the signal strength and thus resolution, was marginal. Consequently, a new camera was purchased via the project and installed (along with the construction of neutron shield to minimize related noise on the diagnostic signal during DIII-D operation).

The raw data from the FTS system is analyzed by a detailed procedure developed that is applicable to the DIII-D system. The data is a line-integral along the line-of-sight that must be inverted to obtain a useful signal. The typical geometry, diagnostic view, and parameters for discharge 142613 considered below are shown in Fig. 1.

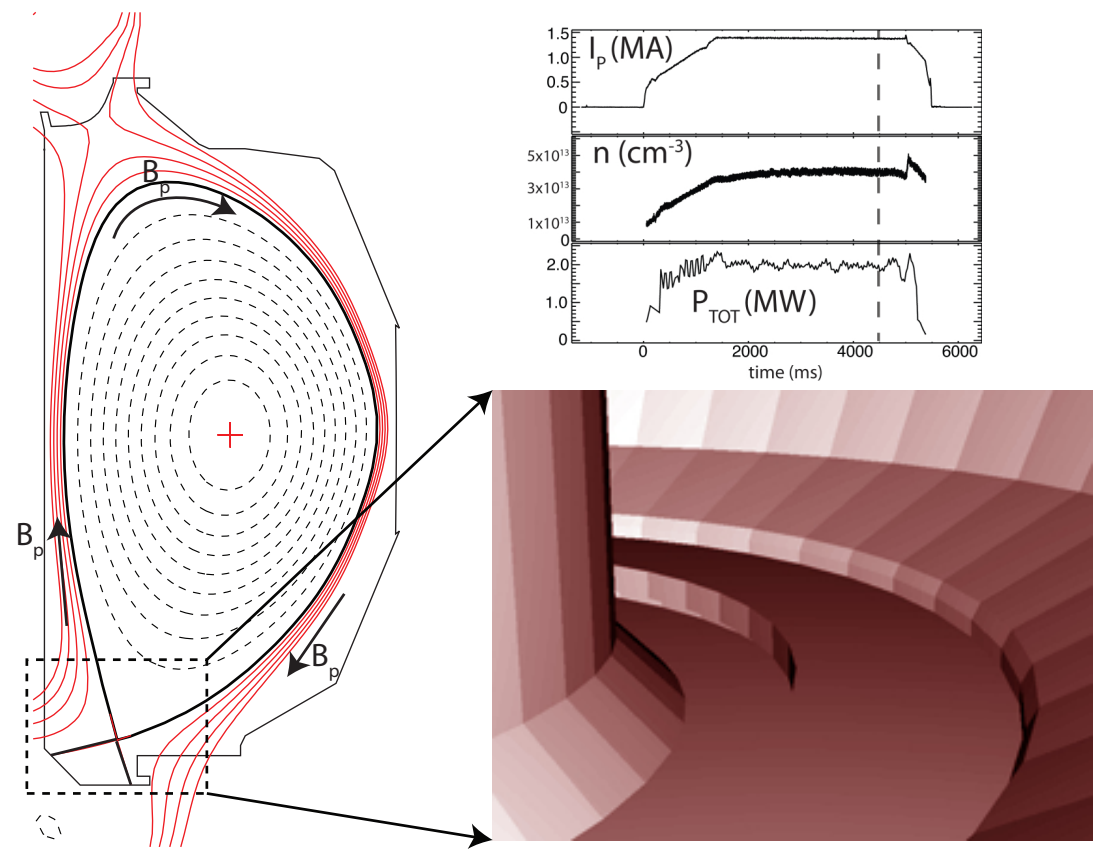

Fig. 1. Geometry of DIII-D discharge (left frame) showing poloidal magnetic flux surfaces and details of the surrounding material walls. The FTS diagnostic view is within the dotted box looking primarily in the toroidal direction; the $3 D$ perspective view is given in the lower-right frame. The upper right frame shows the discharge current, plasma density, and input power. 
The initial raw camera data is simply an image of the $\mathrm{C}^{+2} 465 \mathrm{~nm}$ emission line, modulated by an interference pattern created from the birefringent crystals [1]. From the phase of this interference fringe pattern, the precise center wavelength of the line is locally determined at each point on the image. This wavelength information is related to a Doppler shift of the $\mathrm{C}^{+2}$ line, and with it a carbon ion flow velocity towards the camera. This "flow image" represents a line-averaged measurement of the $\mathrm{C}^{+2}$ flow velocity towards (or away from) the camera, weighted by the $\mathrm{C}^{+2}$ emissivity. Given precise knowledge of the camera location and magnetic geometry, the flow inside the plasma is tomographically reconstructed from the flow image [2-3]. This final reconstruction represents a poloidal cut of the $\mathrm{C}^{+2}$ flow in the plasma. The reconstruction assumes toroidal symmetry, and that all ion velocities are directed along the magnetic field. Considerable care and effort were required to account for the geometrical complexity of the DIII-D vacuum vessel to eliminate spurious signals from reflections, so that the lineintegrated signals can be properly interpreted in a systematic manner; this procedure now seems well in hand for future measurements.

The FTS experiments on DIII-D were made possible by the LDRD purchase of a 14bit Phantom camera with 1000 frame-per-second speed in 2009; as mentioned previously, this camera requires a neutron-shield box. At the beginning of 2011, a different radiation-hardened, 8-bit resolution CIDTEC camera become available at DIII-D that has a built-in image intensifier; this camera was used for the 2011 data acquisition. For the planned 2012 DIII-D experimental campaign, a separate image intensifier usable on either camera should be available to further improve the signal-to-noise ratio.

\section{Analysis of UEDGE transport simulation algorithms}

The inclusion of cross-field particle drifts in UEDGE and other edge transport codes [5-8] often creates convergence difficulties. Recently, a modified form of the drift terms was proposed [8] to improve the numerical behavior of such terms. This technique adds an extra term for the cross-field plasma fluxes that has zero net divergence, and thus is mathematically equivalent to the original terms occurring inside of divergence operators in the transport equations. This approach was implemented and tested within UEDGE. It was found that such a re-formulation gave at best only a modest improvement to the UEDGE ability to convergence, likely because UEDGE, unlike other transport codes, already uses a fully implicit nonlinear Newton-Krylov method to solve the system of transport equations. [6-7]

The numerical analysis did clarify that a key issue is the accuracy of the scheme and the related numerical diffusion. If was found that one could trade off using a low-order upwind numerical scheme having its intrinsic numerical diffusion or use the higher-order central difference scheme but selectively adding an explicit diffusion term to ensure stability. This work was not fully utilized owing to the departure of the postdoc involved, but laid the groundwork for a more optimized UEDGE [6-7]

\section{Comparison of impurity concentration and flow data with UEDGE}

Data from several discharges have been used for comparisons with UEDGE simulations to help validate the UEDGE model of cross-field drifts. The first of these was to complete a set of earlier comparisons between UEDGE and DIII-D data begun just

before this project for discharges with argon gas injection whose concentration can be 
determined from a previously existing diagnostic for argon line radiation. This comparison comprised two companion discharges (\#125850 and 126894), both of which were near double-null divertors (geometrically close to having up/down symmetry), but where one discharge (126894) had a positive toroidal magnetic field, $\mathbf{B}_{t}$, given the ion $\nabla \mathrm{B}$ drift toward the lower divertor plate, and the other having an oppositely directed $\mathbf{B}_{\mathrm{t}}$ and thus upward ion $\nabla \mathrm{B}$ drift. This change in $\mathbf{B}_{\mathrm{t}}$ changes the signs of all the cross-field drifts mentioned early. The amount of argon concentration that was detected in the core region for the two cases differed substantially, and this change can be explained by the flow patterns of argon in the SOL as demonstrated by UEDGE simulations [9]. This work provides important validation evidence that the model for cross-field drift effects on impurities in UEDGE is a major mechanism operative in tokamak edge plasmas.

The new FTS system developed under this project allows us to make a direct comparison between the impurity flow velocity measured and the UEDGE simulation. This validation work is illustrated by discharge 142613, whose characteristics are shown in Fig. 1. The flow velocity of doubly ionized carbon $\left(\mathrm{C}^{+2}\right)$ is deducted from the FTS data using the procedure described following Fig. 1 above, and in Refs. 2-3. The most basic quantity to compare is the spatial distribution of the $\mathrm{C}^{+2}$ emissivity which depends on both the concentration of the $\mathrm{C}^{+2}$ and the electron temperature, $\mathrm{T}_{\mathrm{e}}$; the $\mathrm{T}_{\mathrm{e}}$ dependence of the emissivity is quite nonlinear and peaks around $8 \mathrm{eV}$. Note that UEDGE computes the $\mathrm{T}_{\mathrm{e}}$ profile given a fitting procedure at the outer midplane. Both the data and UEDGE simulation (labeled theory) show two main features in Fig. 2; on the outer divertor to the right of the dotted magnetic separatrix, the emission peaks about the raised shelf region that overlays the duct to the cryopump (at $\mathrm{R} \approx 1.4 \mathrm{~m}$ ) indicating an "attached" plasma with $\mathrm{T}_{\mathrm{e}}$ in excess of $5 \mathrm{eV}$, whereas in the inner divertor leg (left of the dotted separatrix), the emission is localized well off the plate (shown by the angled solid line), indicating a
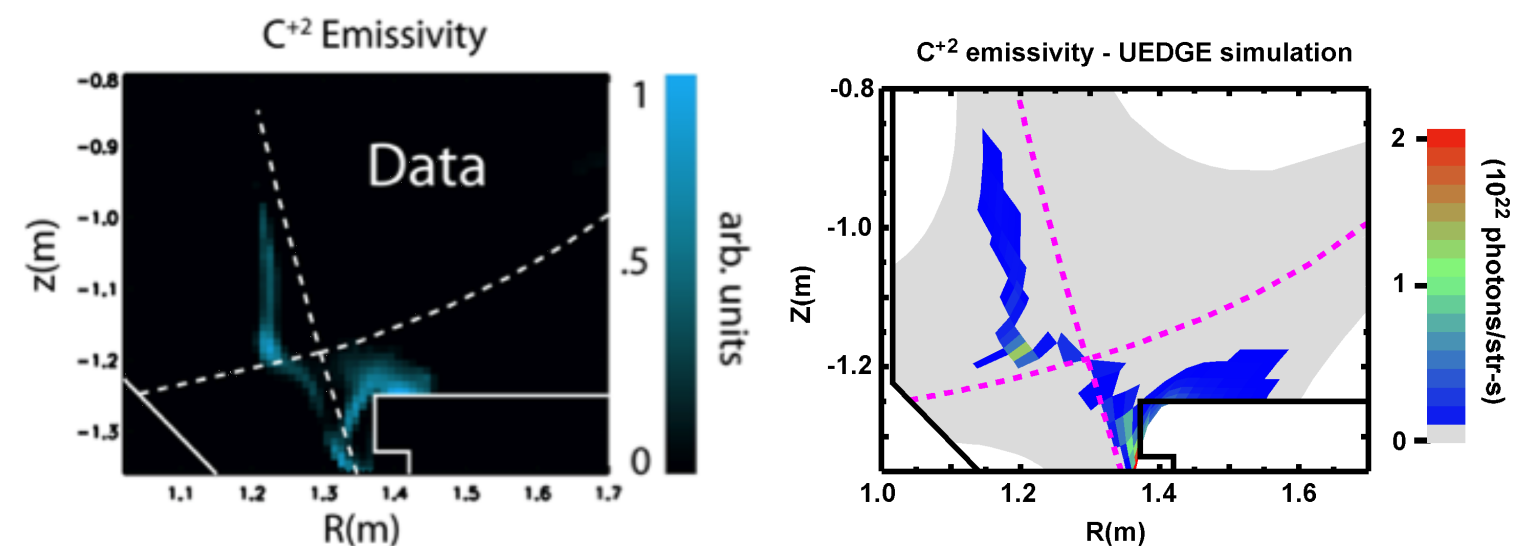

Fig. L. Comparlson of C - emissivity obtainea from the new F IS diagnostlc on VIII-

$D$ discharge 142613 and that obtained from the UEDGE simulation.

cold, detached inner divertor plasma.

The flow velocity of the $\mathrm{C}^{+2}$ is obtained from further processing of the same signal that gives the emissivity. Here the relative strength of the signal cancels out in the analysis. A comparison of the experimental and UEDGE flow velocities are shown in Fig. 3 [4]. There is a substantial agreement in the overall flow velocity magnitude and direction; the velocity is largest above the outer divertor surface and is directed into the 
surface. In the inner divertor leg, the flow changes sign in both experiment and simulation, and the color bar scale for the UEDGE result is set by the minimum/maximum of the UEDGE velocity. There are some notable local differences in the magnitude of the experimental and UEDGE flow, sometimes varying by a factor or 2 or more. There is a systematic inward spatial shift of the UEDGE flow profile on the inner divertor, which likely represents a similar shift in the region where the UEDGE electron temperature is $\sim 8 \mathrm{eV}$ giving the strongest $\mathrm{C}^{+2}$ signal. The experimental signals are average from $\sim 100 \mathrm{~ms}$, which illustrates that the signals themselves are still somewhat noisy; a new image intensifier will be used in future experiments to improve the signal resolution.

We have also used comparison with the FTS carbon flow data to validate the large difference expected in the flow velocity when the toroidal magnetic field changes sign. Two discharges (\#'s 141170 and 142220) were selected that had the same discharge parameters, but where the direction of $\mathbf{B}_{\mathrm{t}}$ is reversed. Beyond the argon case described earlier and published [9], the experiment/modeling comparison is now able to compare directly with flow pattern measured by the new FTS system. Both the data and the UEDGE simulation show that reversing the toroidal magnetic field results in a reversal of

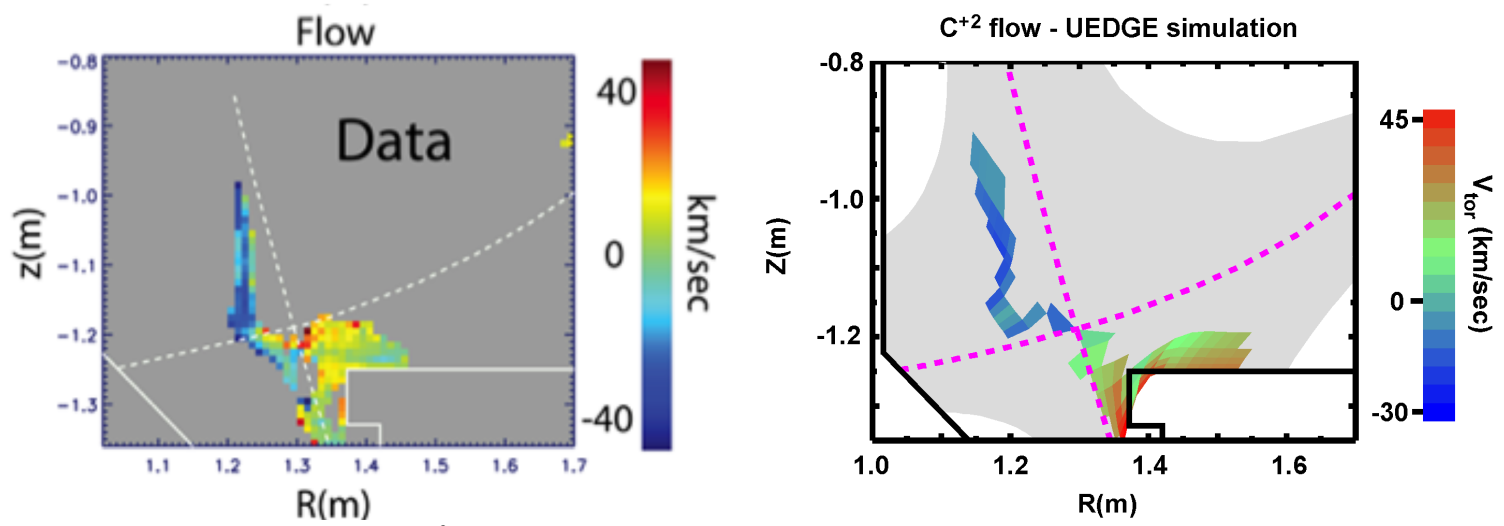

Fig. 3. Comparison of $\mathrm{C}^{+2}$ toridal velocity obtained from the new FTS diagnostic on

DIII-D discharge 142613 and that obtained from the corresponding UEDGE simulation.

the carbon flow velocity. Within the UEDGE model, the flow reversal is expected because the impurity ion velocity is assumed to be directed into the material divertor plates, and this direction is determined by the sign of the toroidal field when the poloidal magnetic field is held constant as in these experiments. The comparison shows that the new flow diagnostic confirms this assumption by provide direct impurity velocity information that was hitherto unavailable and also additional evidence in the validation of the UEDGE plasma transport model.

\section{Conclusions and impact}

The main goals of the project were accomplished, i.e., a new prototype Fourier Transform Spectrometer (FTS) diagnostics was installed on the DIII-D tokamak and used to measure the flow of impurity ions for a series of discharges. These results were then shown to compare reasonably well on the gross features of the impurity flow with the $2 \mathrm{D}$ 
UEDGE edge transport code that evolves densities, velocities, and thermal energies of the divertor plasma and neutrals given plasma profiles measured at the outer equatorial midplane. The quantitative comparison of the detailed flow velocity structure still shows sizeable differences, and improved signal-to-noise upgrades are underway. The importance of the plasma cross-magnetic-field drift velocities on impurity flow in the SOL has been established by comparison of UEDGE simulations with earlier argon injection experiments and are also included in the UEDGE comparisons with the carbonbased measurements from the FTS system.

The numerical algorithms for the cross-field drift velocities in UEDGE code were analyzed in detail. The algorithm suggested by Rozhansky [8] was implemented and tested. While this algorithm did not substantially improve the range of convergence for UEDGE as had been originally hoped, the process of this work led to the understanding that the cross-field drifts add a substantial component of numerical diffusion into the problem. Various schemes to reduce the unwanted numerical diffusion have been implemented and tested, with a hyper-diffusion model being the most promising.

The use and development of the FTS system has now become an important tokamak diagnostic that will continue at DIII-D under base-program funding. In particular, a separate image intensifier that can be used on any camera is planned for use on a new midplane-port view of the plasma (compared to the divertor view shown in Fig. 1) to obtain an improved signal-to-noise ratio for better spatial resolution. For outside funding, we (S. Allen) joined with J. Howard from ANU in 2010 to obtain Australian funding for further development of the FTS system and importantly, its application to ITER. The Australian proposal has yet to be funded, but recently we have also received an expression of interest from researchers at the Joint European Torus that is just beginning operation after a multi-year upgrade to install an ITER-like wall and divertor. All of these follow-on activities would not have been possible without the support and positive results obtained by the FTS system on DIII-D through this LDRD project. 
References (* denotes those papers/presentations from this project; such published papers are attached)

1. J. Howard, "High-speed high-resolution plasma spectroscopy using spatial-multiplex coherence imaging techniques," Rev. Sci. Instrum. 77 (2006) 10F111-1.

2. *J. Howard, A. Diallo, M. Creese, B.D. Blackwell, S.L. Allen, R.M. Ellis, W. Meyer, M.E. Fenstermacher, G.D. Porter, N.H. Brooks, M. E. Van Zeeland, and R.L. Boivin, "Doppler coherence imaging and tomography of flows in tokamak plasmas," Rev. Sci. Instrum. 81 (2010) 10E528 (LLNL-JRNL-514572).

3. *J. Howard, A. Diallo, M. Creese, S.L. Allen, R.M. Ellis, W. Meyer, M.E. Fenstermacher, G.D. Porter, N.H. Brooks, M.E. Van Zeeland, and R.L. Boivin, "Coherence imaging of flows in the DIII-D divertor," Contrib. Plasma Phys. 51 (2011) 194 (LLNL-JRNL-514591).

4. *T.R. Weber, S.L. Allen, D. Hill, W.H. Meyer, G.D. Porter, and J. Howard, " $\mathrm{C}^{+2}$ flow measurements at DIII-D using a coherence imaging spectrometer," oral presentation at APS-DPP Annual Meeting, Salt Lake City, UT, Nov. 14-18, 2011.

5. *T.D. Rognlien, K. Bodi, R.H. Cohen, A.M. Dimits, M. Dorr, S.I. Krasheninnikov, S.K. Nam, D.D. Ryutov, M.V. Umansky, and X.Q. Xu, "Advances in understanding tokamak edge/scrape-off layer transport," IAEA Fusion Energy Conf., Daejeon, Korea, Oct. 11-16, 2010 (LLNL-POST-458732).

6. *S.K. Nam and T.D. Rognlien, "Cross-magnetic-field drift algorithms and results for edge-plasma transport," Int. Conf. Plasma Surfaces Interactions, San Diego, CA, May 24-28, 2010 (LLNL-POST-458732).

7. *S.K. Nam, G.D. Porter, T.D. Rognlien, S.L. Allen, and J. Howard, "High-resolution numerical schemes for cross-magnetic-field drift in UEDGE and comparison with impurity flow measurements, APS-DPP Annual Meeting, Chicago, IL, Nov. 8-12, 2010 (LLNL-POST-461720).

8. V. Rozhansky, E. Kaveeva, P. Molchanov et al., "New B2SOLPS5.2 transport code for H-mode regimes in tokamaks," Nucl. Fusion 49 (2009) 025007.

9. *G.D. Porter, T.W. Petrie, T.D. Rognlien, and M.E. Rensink, "UEDGE simulation of edge plasmas in DIII-D double null configurations," Phys. Plasmas 17 (2010) 112501 (LLNL-JRNL 421320). 